\title{
Comparison of skeletal muscle index-based formula and body surface area-based formula for calculating standard liver volume
}

\author{
Shin Hwang, Geunhyeok Yang, Gi-Won Song, Dong-Hwan Jung
}

Department of Surgery, Asan Medical Center, University of Ulsan College of Medicine, Seoul, Korea

Background: Formula-derived standard liver volume (SLV) has been clinically used for living donor liver transplantation and hepatic resection. The majority of currently available SLV formulae are based on body surface area (BSA). However, they often show a wide range of error. Skeletal muscle index measured at the third lumbar vertebra level (L3SMI) appears to reflect lean body mass. The objective of this study was to compare the accuracy of L3SMI-based formula and BSA-based formula for calculating SLV.

Methods: The study cohort was 500 living liver donors who underwent surgery between January 2010 and December 2013. Computed tomography images were used for liver volumetry and skeletal muscle area measurement.

Results: The study cohort included 250 male and 250 female donors. Their age, BSA, L3SMI, and body mass index were $26.8 \pm 8.7$ years, $1.68 \pm 0.16 \mathrm{~m}^{2}, 45.6 \pm 9.0 \mathrm{~cm}^{2} / \mathrm{m}^{2}$, and $21.7 \pm 2.5 \mathrm{~kg} / \mathrm{m}^{2}$, respectively. The BSA-based SLV formula was "SLV $(\mathrm{mL})=-362.3+901.5 \times B S A\left(\mathrm{~m}^{2}\right)\left(\mathrm{r}=0.71, \mathrm{r}^{2}=0.50, \mathrm{P}<0.001\right)$ ". The L3SMI-based SLV formula was "SLV $(\mathrm{mL})=471.9+14.9 \times \mathrm{LSSMI}\left(\mathrm{cm}^{2} / \mathrm{m}^{2}\right)$ $\left(r=0.65, r^{2}=0.42, P<0.001\right)^{\prime \prime}$. Correlation coefficients were similar in subgroup analyses with 250 male donors and 250 female donors. There was a crude correlation between L3SMI and body mass index $\left(r=0.51, r^{2}=0.27, P<0.001\right)$.

Conclusions: The results of this study suggest that SLV calculation with L3SMI-based formula does not appear to be superior to the currently available BSA-based formulae.

Corresponding author: Shin Hwang

E-mail: shwang@amc.seoul.kr

(c) The Korean Society for Transplantation

This is an Open Access article distributed under the terms of the Creative Commons Attribution Non-Commercial License (http://creativecommons.org/licenses/by-nc/4.0/) which permits unrestricted non-commercial use, distribution, and reproduction in any medium, provided the original work is properly cited. 